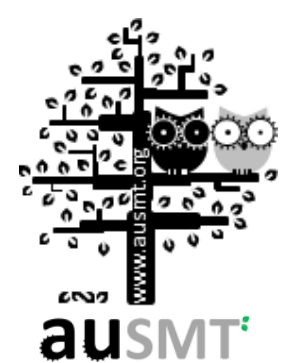

\title{
An Embedded System for Tracking Human Motion and Humanoid Interfaces
}

\section{Ming-June Tsai ${ }^{1}$, Hung-Wen Lee ${ }^{1}{ }^{*}$, Trinh-Ngoc Chau ${ }^{1}$, and Chia-Hong Chao ${ }^{2}$}

\author{
${ }^{1}$ Mechanical Engineering Department, National Cheng Kung University, Taiwan \\ ${ }^{2}$ Electronic Engineering Department, Ming Chuan University, Taiwan \\ (Received 7 February 2012; Accepted 6 June 2012; Published on line 1 December 2012) \\ *Corresponding author: hwlee@mail.ncku.edu.tw \\ DOI: 10.5875 /ausmt.v2i4.144
}

\begin{abstract}
The aim of this research is using embedded CPU to develop a human motion tracking system and construct a motion replication interface for a humanoid robot. In the motion tracking system, we use a CPLD (Complex Programmable Logic Device) which is built in a central control unit (CCU) to generate synchronous signals for all the periphery devices and control the data flow from CCD boards to a PC via a USB chip. An embedded DSP on the CCD board is adopted to control the CCD exposure and conduct image processing. The peak position of exposure was computed by the on-board DSP within sub-pixel accuracy. In the construction of a motion replication interface, the same CCU is used to generate the PWM signals to drive the motors of the humanoid robot. All of the respective firmware coding methods are discussed in this article.
\end{abstract}

Keywords: Embedded System; Human Motion Tracking; Humanoid Robot

\section{Introduction}

In order to meet diverse applications, a variety of embedded CPUs have been developed with the ability to perform particular tasks. In general, the conventional CPU can be divided into two types: CISC (Complex Instruction Set Computer) and RISC (Reduced Instruction Set Computer) [1]. The difference between them depends on the description of the instruction set. A simple categorization of the CPU is shown in Figure 1.

An ASIC (Application-Specific Integrated Circuit) is a special purpose IC customized for a specific application. The development of an ASIC is usually based on a CPLD (Complex Programmable Logic Device) or an FPGA (Field Programmable Gate Array). Using a CPLD or an FPGA, one can alter the firmware during the development of the system. Because the CPLD contains non-vaporized memory like flash ROM and has the ability to time sequence forecast, it is adopted as the main CPU in our motion tracking system.

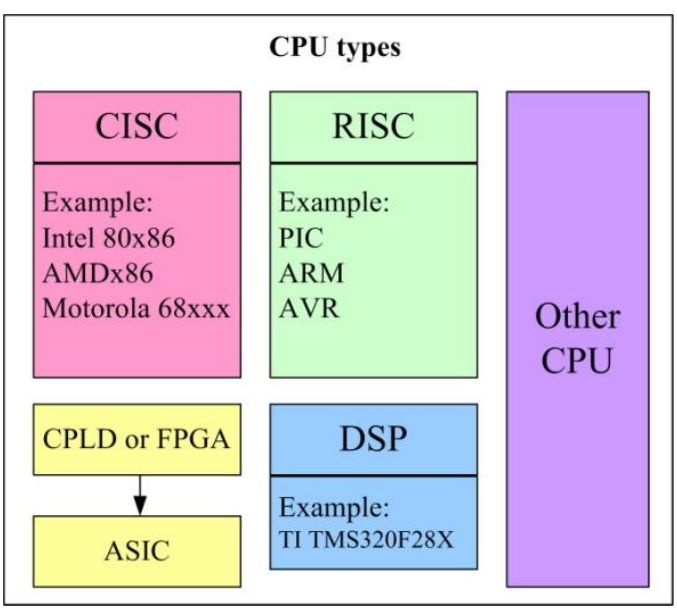

Figure 1. Some CPU Types.

A prototype human motion tracking system named "V2000" has been developed. Inside the motion tracker, there are three circuit boards: a CCU (Central Control Unit) board, a CCD board, and a LED driver board. They are all constructed by using embedded CPUs. The CCD board takes a DSP (Digital Signal Processor) as CPU; 
whereas the CCU and LED driver boards both take CPLD chips but with different specifications. Since the system captures LED lighting images by linear CCD, it is efficient to use the DSP for real-time image processing.

The CCU board is also used in the control of the humanoid robot. Once the USB chip receives the joint angle commands from the PC, an 8051 processor inside the USB chip will convert the serial data into parallel form and transmit them to the CPLD. Then a timer will start to count until the corresponding PWM signal is generated for each motor of the humanoid robot. Finally, all the PWM signals will be simultaneously sent to drive the motors to perform the motion.

The main purpose of this research is to describe how the embedded system is built. The V2000 motion capture system has been verified to have the ability to capture 32 LED markers continuously. The control board we use can distribute up to 32 PWM signals for the servo motors of the humanoid robot in response to commands generated by a PC. The problems of how the human motion is tracked by the system and how the humanoid robot replicates the motion are subjects of our future studies.

\section{Human Motion Tracking System}

The motion tracking system consists of three sensor units. Each unit is equipped with one cylindrical lens and one linear CCD. A total of three sensor units are fixed on a frame. The orientation of the middle sensor is fixed horizontally; whereas the other two units are placed on a vertical plane. Such an arrangement causes the three generating planes to meet at a point. The inclination angle between these two side units is eleven

Ming-June Tsai is a professor in the Department of Mechanical Engineering, National Cheng Kung University. He received a Ph.D. degree in Mechanical Engineering at Ohio State University in 1986. He teaches courses in mechanical design, machine vision, and robotics. His current research focus is on humanoid robots, human body modeling, and the capture of body performance motion.

Hung-Wen Lee is a postdoctoral fellow in the Department of Mechanical Engineering, National Cheng Kung University. He received a Ph.D. degree in Mechanical Engineering at National Cheng Kung University in 1993. His research interests are in human body modeling and motion capture systems.

Trinh-Ngoc Chau is a Ph.D. candidate in the Department of Mechanical Engineering, National Cheng Kung University. He received a B.S. degree in electronic engineering from the Ho Chi Minh City University of Technology, Vietnam, in 1997, and a M.S. degree in mechatronics technology from Southern Taiwan University in 2007. His research interests include embedded system design, vision control, and sign language processing for the hearing-impaired.

Chia-Hong Chao received his Master of Information Management from Georgia State University in 1982. He is currently a full-time lecturer in the Department of Electronic Engineering of Ming Chuan University. He teaches algorithm and software programming. His research topics are on semantic searching methodology, artificial intelligence, and computer-assisted Instruction. degrees. An optical filter is placed in front of the CCD sensors in order to cut off light with a wavelength of less than $800 \mathrm{~nm}$. The capture range of the motion tracker is about $2000 \times 2339 \mathrm{~mm}$ at a viewing distance of $2 \mathrm{~m}$. Its schematic diagram is shown in Figure 2 . This is the reason it was named the V2000 system. Four modules are fabricated in the same way and placed around the human body in order to reduce lighting occlusion during the process of capturing motion [2].

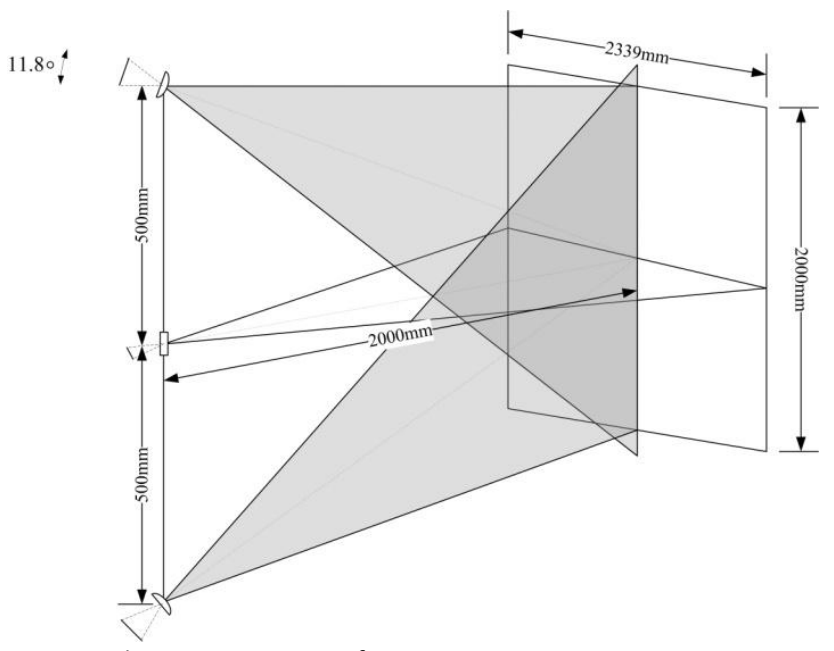

Figure 2. The capturing range of V2000.

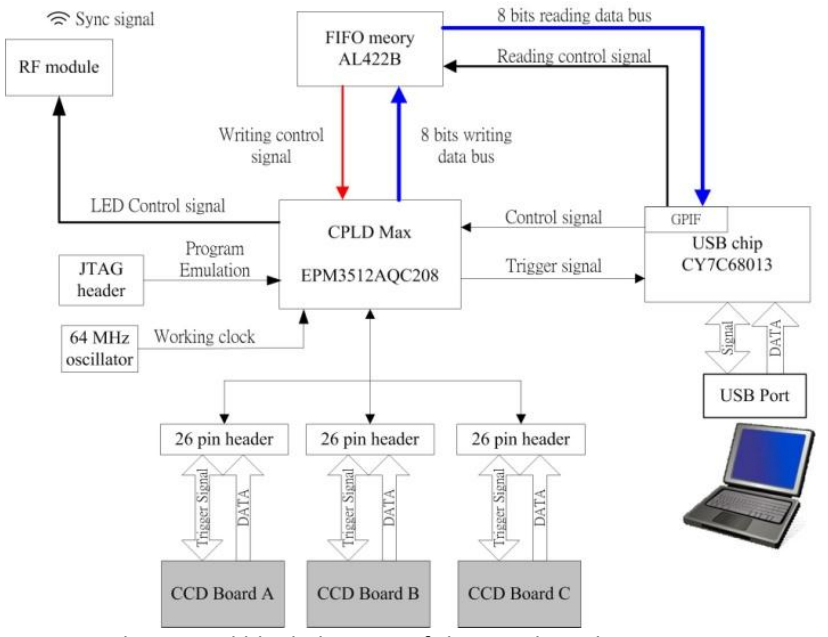

Figure 3. The control block diagram of the CCU board.

\section{CCU Board}

The CCU board consists of three main components: a CPLD by Altera (Max EPM3512AQC208-10) [3], a USB2.0 chip for data transmission by Cypress (FX2 CY7C68013USB) [4], and a FIFO buffer by Averlogic (AL422B) [5]. The control block diagram is shown in Figure 3. The CPLD will first receive the start signal from a $\mathrm{PC}$, and then send synchronous trigger signals to a DSP to control the CCD units designed for capturing images. 
After capturing the images, the CCD unit will return the image peak values to the FIFO on the CCU, and transmit the data to the PC via a USB interface.

The CCD board consists of a TI TMS320F2810 DSP used to control the CCD [6], a SONY ILX-554B linear CCD [7], and an $A D C$ by Analog Device (AD9220) for converting the image peak signal [8]. The LED driver board consists of an ALTERA Max EPM3064A CPLD to control two LED driver chips (Toshiba TB62706). It can flash up to 32 LEDs in many combinations.

As shown in Figure 4, the CCD chip needs ROG and CLK signals to control the exposure for capturing images. Since the duty cycles of both signals are not $50 \%$, the CPLD generates the corresponding PWM signals for them. Two signals, ROG_1 and ROG_2, generated by the CPLD are shown in Figure 5, in which ROG_1 (ROG) is for the exposure time signal and ROG_2 is combined with a $2 \mathrm{MHz}$ clock as the CLK signal for the three CCD units. Figure 5 also shows an example of VHDL (Very High Definition Language) [9] code for the CPLD to generate the signals written by [10].

\section{CCD Unit}

Because any spatial marker position has three dimensional coordinates, three CCD units are needed for a motion tracking set to establish a mathematical model. For each CCD unit, the light emitted from the LED marker passing through a cylindrical lens will focus on the plane that will intersect with the linear CCD sensor. Therefore, the intersecting point together with the axis of the cylindrical lens will define a plane. The light intersecting point on the linear CCD sensor will have the highest intensity of illumination due to lens focus. To ensure that the change of the plane's orientation due to the marker movement can be detected, the linear array CCD sensor should be placed perpendicular to the optical axis of the cylindrical lens. After each plane defined by the light projecting point on the linear CCD and the optical axis of the lens is known, the space position of the light marker can be calculated by the intersection of the three planes shown in Figure 6.

The control block diagram for the CCD unit is shown in Figure 7. The control sequence is illustrated in Figure 8. When the CCD units receive the trigger and clock signals from the CCU board, the linear CCDs will start to capture the image of the lighting markers. Then the captured image signals are sent to the OP amplifier and the $A D C$ chip to convert the analog signals into digital form. Finally, the image data will be processed by the DSP chip to find the peak position in sub-pixel level and be transmitted to the CCU board [11].

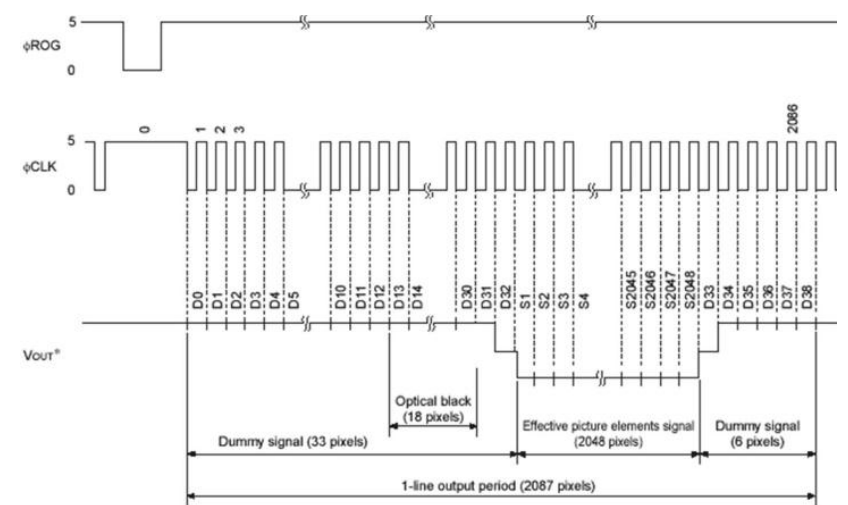

Figure 4. ROG and CLK signals for ILX-554B [6].

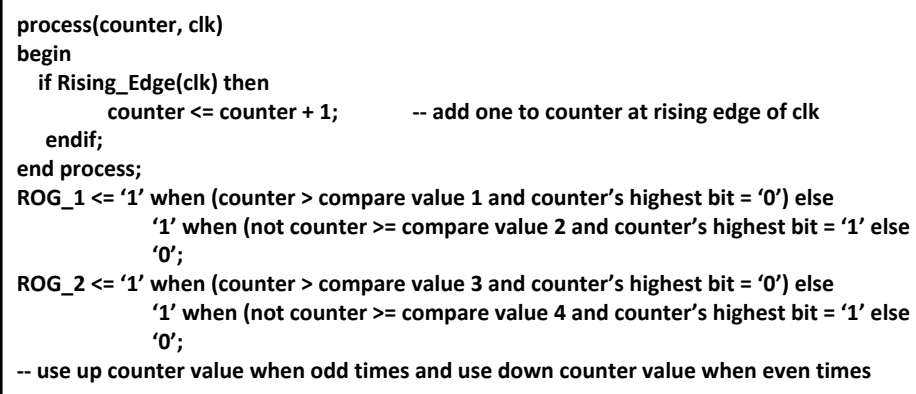

ROG $1<=$ ' 1 ' when (counter $>$ compare value 1 and counter's highest bit = ' 0 ') else ' 1 ' when (not counter $>=$ compare value 2 and counter's highest bit $=$ ' 1 ' else ' 0 ';

ROG_2 < = ' 1 ' when (counter > compare value 3 and counter's highest bit = ' 0 ') else ' 1 ' when (not counter $>=$ compare value 4 and counter's highest bit = ' 1 ' else ' 0 ';

-- use up counter value when odd times and use down counter value when even times

Figure 5. VHDL code for the CPLD [10].

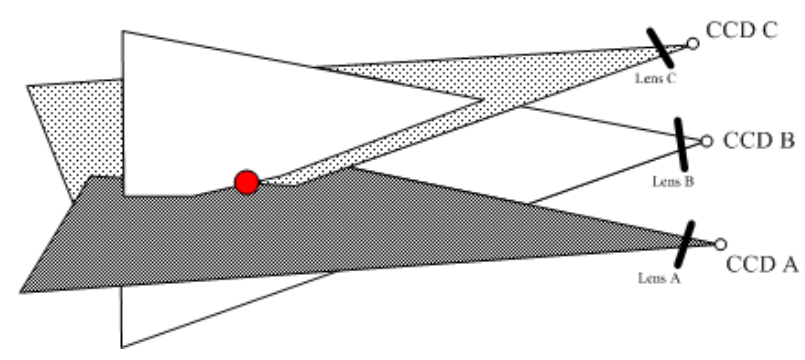

Figure 6. To locate the spatial coordinates of a point.

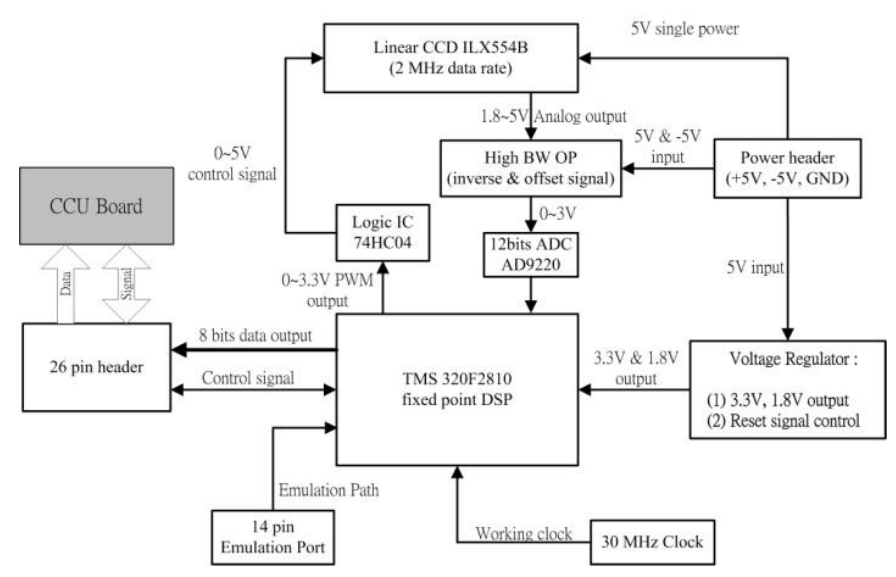

Figure 7. The control block diagram of CCD unit. 


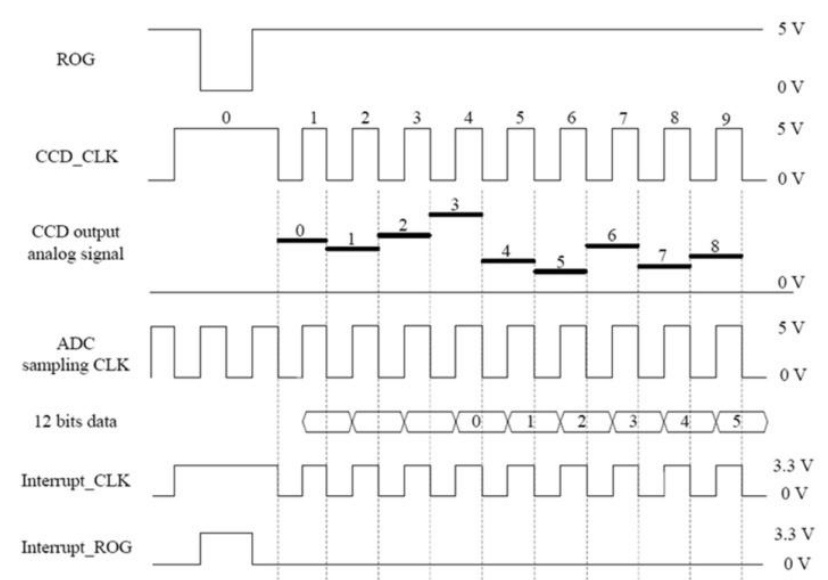

Figure 8. The time sequence of CCD unit [8].

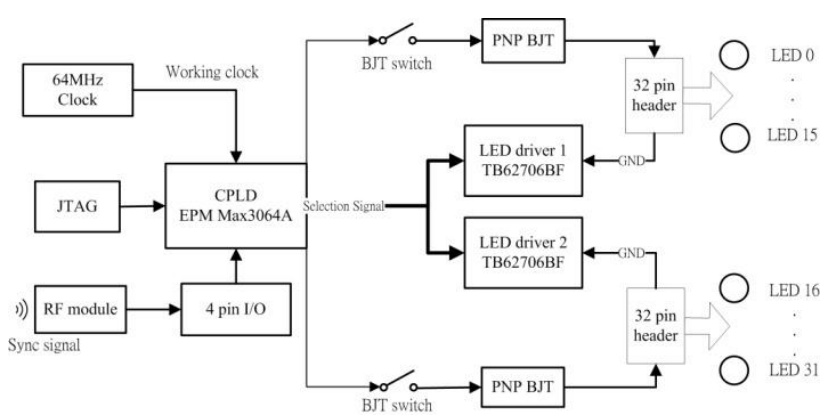

Figure 9. The control diagram of the LED driver board.

\section{LED Driver Board}

The LED driver board has another smaller CPLD to control two LED driver chips TB62706BF [12]. The CPLD was programmed so that it can flash up to 32 LEDs in many combinations. The control block diagram is shown in Figure 9. When the control signal from the CCU board is received, the CPLD will control the flashing of the LEDs.

The LEDs should be lighted in sequence because the linear CCD can only capture one marker at one time, or else we cannot distinguish which LED is the target. The flashing of the LEDs and the exposure of the CCD should occur simultaneously. Hence, the signals from CCU have to trigger the CCD units and the LED driver board at the same time.

The ROG synchronization signal from the CCU board (The frequency of the ROG signal is the same as the ROG_1 signal) will command the LED driver to send a flash signal to the LED. The Data signals will increase one by one to flash each LED in order. This is also called a one-shot circuit.

\section{System Calibration}

Once the system is set up, it is necessary to have a calibration before motion capturing can begin. An optimization process is employed in the calibration process to increase the system's accuracy. This process will modify the geometrical parameters by comparing the ideal positions with captured (computed) positions.

A precision metal tower that contains 16 LED markers is mounted on an $X-Y$ linear stage. As shown in Figure 10, the tower moves $100 \mathrm{~mm}$ incrementally at each step. The whole capturing range is about 1000x900x400 mm (Height-Width-Depth). The tower motion was controlled by another CCU board. Since the precision of the marker position is very important in this process, the two linear stages are also equipped with optical linear encoders. As a result, eight hundred LED markers are captured by moving two linear stages and switching LEDs on the tower at predefined positions. The data collection process is done automatically with a single command given through the PC. To make sure that the LED tower is not shaking during the capturing process, the image capturing should wait for several seconds after each movement. During the calibration process, the motion tracking module and the linear stages are all placed on a vibration-isolated optical table.

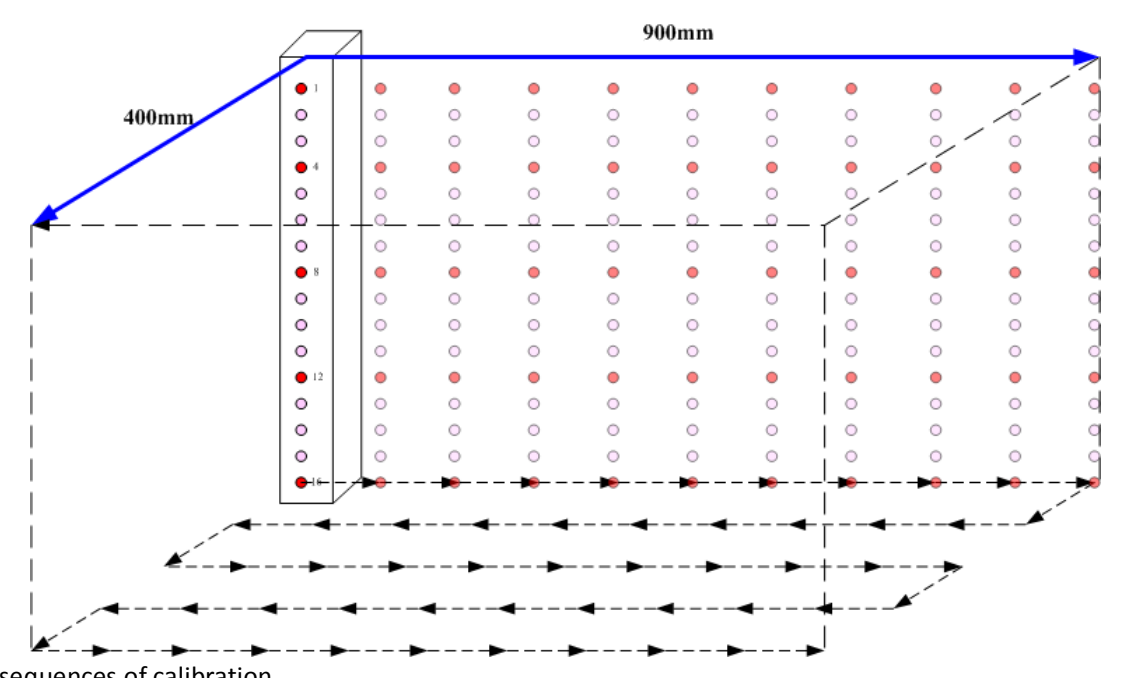

Figure 10. The capturing sequences of calibration. 


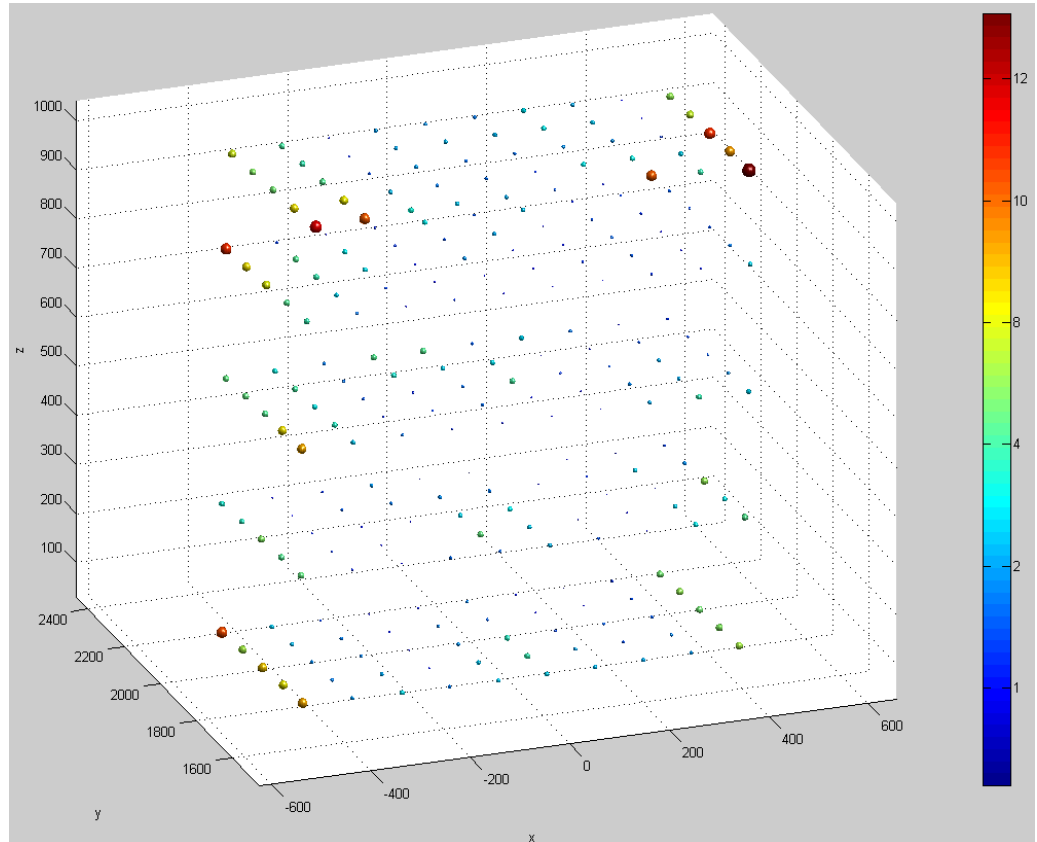

Figure 11. The bubble plot of error distribution for V2000.

The calibration errors before the optimization are listed in Table 1 . The calibration errors after the optimization are listed in Table 2. The mean error in 3D is improved from $6.633 \mathrm{~mm}$ to $4.120 \mathrm{~mm}$, i.e. about a $38 \%$ reduction of the error. It is also obvious that the system is more precise and stable after the optimization.

Table 1. The calibration errors before optimization.

\begin{tabular}{|ccccc|}
\hline Unit: $\mathrm{mm}$ & $X$ & $Y$ & $Z$ & 3D \\
\hline MEAN & 1.801 & 6.198 & 1.529 & 6.633 \\
\hline MAX & 10.160 & 47.73 & 12.457 & 50.365 \\
\hline STDEV & 2.269 & 8.762 & 2.153 & 9.304 \\
\hline
\end{tabular}

Table 2. The calibration errors after optimization.

\begin{tabular}{|ccccc|}
\hline Unit: $\mathrm{mm}$ & $\mathrm{X}$ & $\mathrm{Y}$ & $\mathrm{Z}$ & 3D \\
\hline MEAN & 1.213 & 3.687 & 1.382 & 4.120 \\
\hline MAX & 5.814 & 14.060 & 6.494 & 14.390 \\
\hline STDEV & 1.046 & 3.334 & 1.160 & 3.682 \\
\hline
\end{tabular}

Figure 11 shows a bubble plot of the error distribution. The error is smaller than the system resolution due to the contribution of a peak position sub-pixel computation conducted by the DSP [11]. The errors are bigger around the sides of the capturing range. This is usually caused by the clearance/deformation of the mechanical parts and the inaccuracy of the stage motion.

\section{The Humanoid Control}

A humanoid robot with 29 degrees of freedom was designed for the replication of human motion. Figure 12 shows the robot named "Sunny." In general a great many

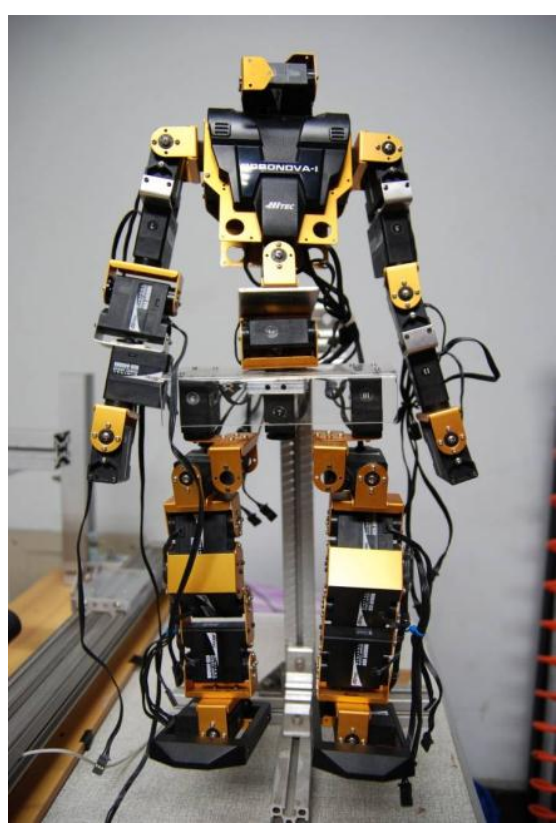

Figure 12. The humanoid robot.

degrees of freedom are needed in a humanoid robot in order to duplicate the complexities of human motion. Since this human motion tracking system is designed to capture larger human joint motions, relatively smaller finger motions will not be captured. The motors chosen for the Sunny robot are HSR-8498SB servo motors [13]. The PWM signal needed to control the motor position is shown in Figure 13.

\section{-95deg -60deg Centre +60deg +95deg

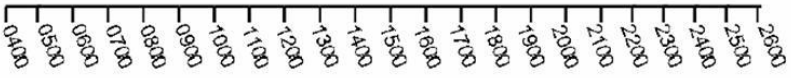 Micro seconds}

Figure 13. The corresponding PWM signal.

\section{PCI Interface}

Because the CPLD on the CCU board can also generate corresponding PWM signals for the motors, the CCU board can be used to control the robot. A PC interface has been developed for users to control or teach the robot by typing or dragging the slider bar to set the rotation angle for each servo. There are two teaching versions: an offline control version and an online version. The motion range of each motor is about 180 degrees. The offline mode means that users can set every servo before performing the motion, and the online mode means when the user changes the angle value of any motor, the motor will rotate simultaneously. The interface shown in Figure 14 is for the online version. 


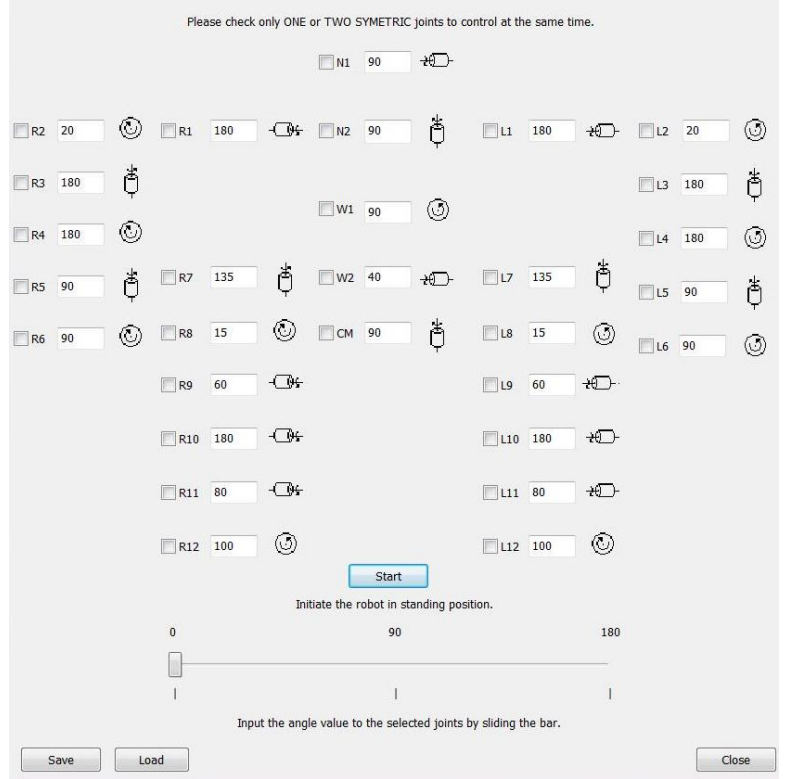

Figure 14. The PC interface (online).

\section{USB Firmware}

A USB chip was installed on the CCU board in order to communicate with a PC. This chip contains an 8051 processor. The resolution of the servo is about 1 degree, so a byte ( 8 bits, $0-255$ ) is enough to record an angle value. A total of 29 bytes (232 bits) data should be transmitted from the PC to the USB, and then to the CPLD. After the USB receives the angle value from the PC, the 8051 processor will convert the USB serial data into parallel form. Finally, 8 bits I/O between the USB and the CPLD are used to send the data. Figure 15 shows part of the firmware code using Keil-C.

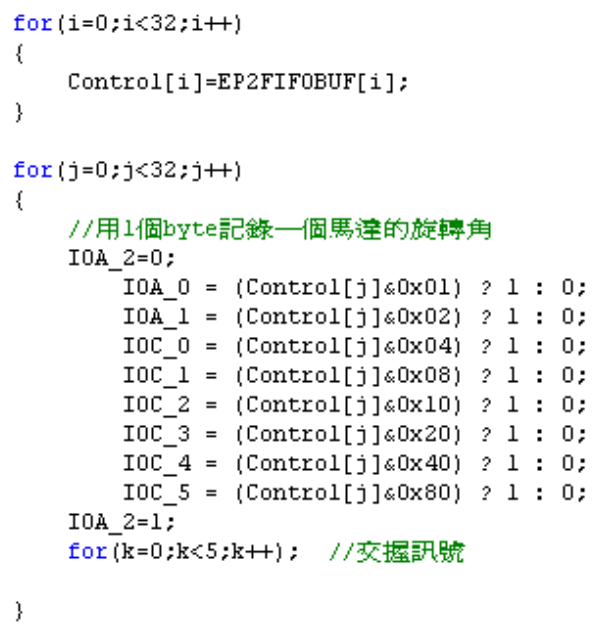

Figure 15. The main code of USB firmware.

\section{CPLD Firmware}

The task of the CPLD on the CCU board is to generate PWM signals for each servo motor. The task is similar to the task of controlling the linear stage during the calibration process of the motion tracking system. On receiving the joint angle values from the $P C$ via the USB chip, the timer will start to count until the corresponding width of the PWM signal has been generated. Finally, all of the signals will be sent to the servos. Figure 16 shows the control flow diagram of the humanoid. The teaching results of two robotic postures are demonstrated and shown in Figure 17.

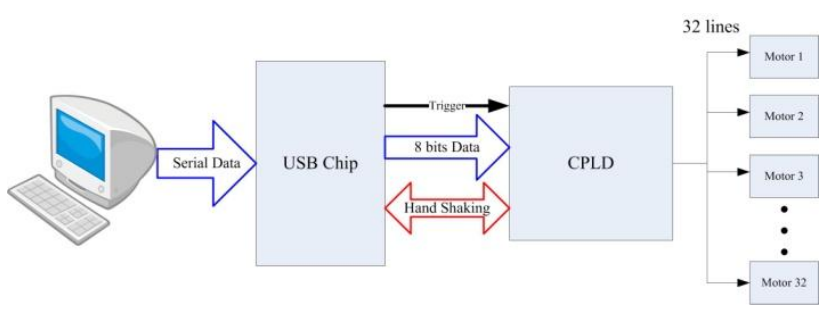

Figure 16.The control flow of the humanoid robot.

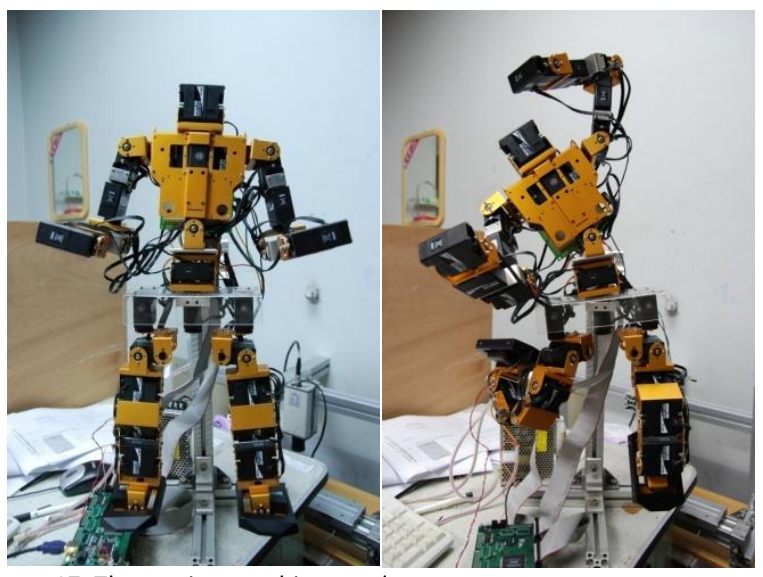

Figure 17. The motion teaching result.

\section{Conclusion}

This article presents a method for using embedded CPUs to build a human motion tracking system and a humanoid replication system. For the motion tracker, a CPLD, a USB chip, and a DSP were used and their respective firmware programs were constructed. An optimization process in the calibration improved the accuracy of the system. For the humanoid motion replication system, the same CCU board was used. An 8051 in USB chip and a CPLD were used to send corresponding PWM waves. The overall goal of this study was to build a completed human motion tracking system. A 3D graphical human motion editing system is under construction.

\section{Acknowledgement}

This work is funded by the National Science Council under grant NSC-96-2221-E-006-275-MY3. 


\section{References}

[1] J. Catsoulis, Designing embedded hardware. Sebastopol, CA: O'Reilly, 2005.

[2] M. J. Tsai, H. W. Cheng, and Y. J. Huang, "Implement a novel human motion tracking prototype system," in The 26th National Conference on Mechanical Engineering, Tainan, Taiwan, 2009.

[3] MAX 3000A programmable logic device family datasheet (version 3.5). San Jose, CA: Altera Corp., 2006.

Available:

http://www.altera.com/literature/ds/m3000a.pdf

[4] EZ-USB $^{\circledR}$ technical reference manual. San Jose, CA: Cypress Semiconductor, 2009.

[5] AL422B data sheet. San Jose, CA: Averlogic Technologies Inc., 2004.

[6] ILX554B data sheet. Tokyo, Japan: Sony Semiconductor, 2002.

[7] TMS32OF2810, TMS320F2811, TMS320F2812, TMS320C2810, TMS320C2811, TMS320C2812 digital signal processors data manual. Dallas, TX: Texas Instruments, 2003.
[8] AD9221/AD9223/AD9220: Complete 12-Bit 1.5/3.0/10.0 MSPS monolithic A/D converters data sheet. Norwood, MA: Analog Device, 2003. Available:

http://www.analog.com/static/imported-files/data sheets/AD9221 9223 9220.pdf

[9] P. C. Tang, VHDL and digital logic design. Taipei, Taiwan: Kao-Li, 2002.

[10] C. F. Kuo, "A study of synchronization of two optoelectronic motion trackers," M.S. Thesis, Department of Mechanical Engineering, National Cheng-Kung University, 2006.

[11] M. J. Tsai, Y. H. Pan, and H. W. Jang, "Mechatronic design of an optical human motion tracking system," in International Symposium on Robotics and Mechatronics (ISRM), Hanoi, Vietnam, 2009.

[12] TB62706BN/BF data sheet. Tokyo, Japan: Toshiba Semiconductor, 2002.

[13] Hitec HSR-8498HB digital servo operation and interface (Rev. 0.5). London, UK: RoboSavvy Ltd., 2007.

Available:

http://robosavvy.com/Builders/i-Bot/HSR8498HB Servo.pdf 